\title{
Thoughts on the Reconstruction of Legal Regulations of Artificial Intelligence-From the Perspective of Surgical Robot
}

\author{
Hongmin Zhang \\ School of Business, Macau University of Science and Technology \\ M.U.S.T Macau, China
}

\begin{abstract}
The rapid development and wide application of intelligent robots have created a more sophisticated and intelligent society. However, countries must pay attention to various social problems hidden safety problems. Supporting legal provisions that keep up with the times should be the first, and the determination of the legal status of intelligent robots is undoubtedly the premise of some specific regulatory measures. Based on the application of intelligent robots in the medical field, providing electronic personality to intelligent robots would be an effective resolution. On this basis, problems such as the rights and legal responsibility, the tort liability would correspondingly get resolved. Intelligent robots undoubtedly help pushed advancement of society, so the development of law cannot just give up eating for fear of choking and should not hinder social progress. In a word, law is more and more important in intelligent society.
\end{abstract}

Keywords-Thought; Reconstruction; Artificial intelligence; Surgical robot

\section{INTRODUCTION}

Artificial intelligence technology since 1955, has experienced more than 60 years of development, is now mainly used in robotics, image recognition, language recognition, expert systems, automatic planning and other fields. With the breakthrough of key technologies such as deep learning and neural network, the concept of 'artificial intelligence + medical treatment' has being raised. It has experienced the development of data integration, data sharing \& perception intelligence and cognitive intelligence $\&$ health data. Current applications and research in the medical field include virtual assistants, medical imaging, drug mining, emergency room/hospital management, health management, mind health, pathology, etc. With effective and accurate improvement on traditional medicine, human has gradually entered the era of personalized medicine.

In May 2016, children's national health system developed the world's first autonomous surgical robot Smart Tissue Autonomous Robot (STAR), which is capable of handling soft tissue. Relative to the da Vinci surgical robot working under the control of a doctor, STAR has transformed itself from an assistant to become the leading role taking over the work of surgeons, providing accurate, efficient and perfect surgical treatment services. However, the robot also has flaws, what should do if the operation fails? What about misdiagnosis? How to identify the responsibility? What about the data loss and privacy leak? At present, the regulation of medical artificial intelligence is still in the blank stage. As the foundation of medical artificial intelligence, medical big data has not been regulated. There are no clear legal instructions on the right to privacy, access to data, privacy standards for medical data, data security, liability norms, and the wrong way for laws to contain innovation. The lack of legal norms torture and also limit the landing of intelligent medical technology.

As Dickens put it, 'This is the best of times and the worst of times'. The artificial intelligence revolution has undoubtedly brought us a more intelligent, refined and humane society. However, the corresponding social problems and security hidden dangers under intelligence are constantly appearing, and the whole world will face the new challenge of intellectualization to legal order.

\section{THE LEGAL STATUS AND RESPONSIBILITY OF} INTELLIGENT ROBOT UNDER LEGAL REGULATIONS

Intelligent medical robots use super algorithm like ant colony algorithm, fuzzy set application, particle swarm optimization algorithm, artificial neural network and so on, supplemented by data mining, case analysis and semantic description models, they have the ability to make independent decisions and deep learning. This has enabled intelligent medical robots to have the basis of consciousness and mind.

Through deep study of massive medical diagnosis data, the surgical robot can improve its diagnostic ability and become an experienced robot doctor, whose autonomous operation behavior is similar to that of doctors, that is, natural persons. But is it a natural person, legal person, animal or even an object in terms of legal status? Therefore, it is very necessary to define the main legal responsibility of artificial intelligence in medical field and provide legal guarantee for the application of artificial intelligence.

This article is sponsored by the Macau Foundation. 


\section{A. Legal personality}

The so-called legal personality refers to the legal qualification of the enjoyment of rights and obligation. In civil law, both natural person and legal persons may have legal personality on the basis of self-awareness. But the traditional machine is only a tool of the individual, the company or other legal body. So, are intelligent robots different from traditional machine? It is reported that in 2007 alone, the Food and Drug Administration of the United States received more than 200 complaints alleging that surgical robots caused burns, cuts and infections on patients. Of these, 89 patients died. Are the cases of human damage caused by a large number of robots caused by technical loopholes or the improper use of intelligent machine managers? Or, does an artificial intelligence system act beyond the original control scheme?

The European Commission of Legal Affairs in 2016, in its draft legislation, designated smart robots as 'electronic person', with specific legal rights and obligations. Electronic personality may be applied in cases where the robot makes its own decision or otherwise interacts independently with a third person. Does this mean that intelligent robots have acquired similar self-consciousness from human knowledge or big data intelligence? If not, there will be a serious conflict in essence with the dichotomy of subject and object in jurisprudence. Since the robot is different from the natural person with life, and also from the legal person of the natural person assembly, it is not suitable to be treated as a legal subject in jurisprudence. In accordance with the logic of the international community to deny the legal personality of human cloning on the basis of disturbing the ethics of human consanguinity, intelligent robots also have the risk of counteracting human society. Whether it can also deny its legal personality? Since it has no legal personality, its legal status is still equivalent to the ordinary machine.

However, intelligent robots, after all, are different from ordinary machines that do not have the ability to think and communicate, and it is obvious that their rapid development has forced the law to accept the view of electronic person. The intelligent robot is regarded as a special thing between legal person and tool, which has the legal subject qualification as well as the attachment in the tool property.

\section{B. Rights and legal responsibility}

The change in the EU legal affairs commission's understanding of the subject of artificial intelligence is intended to clarify the legal status of intelligent robots, which is the premise of defining the rights and obligations of intelligent robots, and that is clearing the way for on solving the problem of intelligent robot tort liability, data right and privacy protection.

Under the civil law system, the capacity of rights is regarded as the legal status or qualification of natural persons to act as civil subjects, enjoy civil rights and undertake civil obligations. Once the intelligent robot is recognized as a legal personality, it is equal to the right ability, and can independently express its meaning and claim the general civil rights under an independent legal personality, including personal and property rights. At the same time, legal responsibility is also required. As intelligent robots act independently in more and more scenarios, although humans design, manufacture and deploy them, their behavior is not subject to direct human instruction, and the stronger their autonomy is, the more difficult it is to treat it as a simple tool in the hands of other subjects, and the more dangerous the unpredictable behavior it makes on its own. And the legal risk and harm that result from this must have the main body that bears responsibility. Although artificial intelligence can act independently, its ability to bear the consequences of behavior is limited, which makes the legal personality of intelligent robot limited. It is worth noting that the limitation on legal personality of artificial intelligence will not absolve its responsibility. But the existing liability rules are clearly inadequate, and new rules are needed to address how a robot can be partly or wholly responsible for its actions or omissions.

\section{Tort liability}

Traditionally, medical tort is that medical institutions and medical personnel have committed medical acts that infringe on the legitimate rights and interests of patients and have formed legal consequences. The legal rights and interests of the patients here include the right to health, the right to subsistence and other rights of privacy. Subjective fault, illegal act, damage result, cause and effect are all necessary parts in the process of identification.

How to translate the unstructured data from medical records, images and test reports into the structured data of machine can be used for machine learning and algorithm realization is the foundation of medical artificial intelligence development. In addition, the realization of medical intelligence not only requires the quality of data itself, but also the way of data acquisition. At present, there is a lack of reasonable data sharing and data circulation mechanism. The ownership of medical data is restricted by fuzzy data sharing, data privacy protection and data security. With the development of artificial intelligence in the field of biomedicine, we have to think about the division of tort liability and liability for medical damage caused by artificial intelligence. In March 2017, Google, Google brain and scientists at verily developed an artificial intelligence and pathologist to diagnose breast cancer. The result of the competition was Pathologist accuracy rate is $73.3 \%$, artificial intelligence accuracy rate is $88.5 \%$. Therefore, based on the limitations of existing technology, artificial intelligence can't achieve $100 \%$ accuracy, there is inevitably a misdiagnosis.

While improving the success rate of surgery, surgical robots may directly endanger the health and life of patients because of product design defect, product quality defect, system failure or even unavoidable misdiagnosis. The probability of its occurrence will also increase with the complication of technology. There must be responsibility for the damage. The responsibility arises from the causal relationship between the infringement and the damage suffered by the victim, and the intelligent robot is no exception. There can be no limits on the type and extent of damage that can be compensated, nor on the compensation that the victim may receive because the damage was caused by a smart robot. 
The nature of artificial intelligence remains a tool, and even recognition of its legal status cannot change its attributes that serve the development of human society. The limited legal personality it enjoys implies that damages should be shared by its designers, developers, producers and users. The traditional tort legal system is borne by medical institutions and manufacturers without fault and joint liability. Medical artificial intelligence is more complex than ordinary medical device products. It has more complex algorithms and medical data. And there is no artificial intelligence technology and safety standard in different medical subdivisions, to specify relevant contents such as diagnostic failure rate requirements, disclosure or filing of algorithms, legal review of medical data, periodic inspection or inspection, the product flaw identification of artificial intelligence medical instrument becomes blank.

The tort regulation of intelligent robot mainly focuses on the subject of liability burden and the safety of data use. The existing laws regulating medical damage are mainly the Medical Accident Management Regulations and Tort liability act. In the determination of civil liability, the introduction of new technology makes it more and more easy for doctors to be exempted from liability under the traditional fault identification method, because in the case of intelligent robot operation, doctors no longer have the basis for liability. As for the burden of proof, the tort liability law clearly defines the fault principle of liability for medical is damage, the victims of medical damage under the autonomous operation of the intelligent robot bear the burden of proof for the fault of the robot's behavior. It is undoubtedly more difficult to prove the fault of complex robot than traditional medical damage. Therefore, the introduction of lower standards of burden of proof and the mandatory preservation and provision of complete data records will help to reduce the difficulty of the victim's proof. On the basis of maintaining the existing liability system, some countries provide comprehensive protection to victims by improving the way of tort liability investigation. In addition, under the condition that the safety risk of medical artificial intelligence system is not clear, the signing right of medical diagnosis result of medical artificial intelligence system is applied.

\section{The legal choice of moderate supervision}

Artificial intelligence has fully penetrated the medical field. The application of artificial intelligence in the field of medical treatment is directly related to the safety of users. The data privacy right, right to use, medical data privacy, algorithm discrimination and so on need to have the corresponding law to regulate.

At present, many countries in the world have been involved in artificial intelligence norms, the United States promulgated Health Insurance Portability and Accountability Act (HIPAA), in 1996, Providing for the establishment of a national electronic medical transaction security and supervision system, and regulating medical information security and privacy, Subsequently, the Security and Innovation Act, the Privacy Standards for Personal Recognition Health Information and the Guide to Mobile Medical Applications were promulgated to regulate Internet electronic medical information. The commercial application of medical information must comply strictly with the HIPAA and HITECH provisions. The mandatory regulations in HIPAA require changes in the practices of health-care providers, including the establishment of electronic data interchange (EDI), Standardized mechanisms for security and confidentiality of all health-related data. In addition, patient health records, management records and financial data are standardized formats; Each health-care entity uses a unique authentication code (ID number) to ensure the confidentiality and integrity of information data.

Because of the complex ethical boundary of medical artificial intelligence application, the lack of management will aggravate the risk of uncertainty in application, while too much control will also be a constraint on the development of the industry. Reasonable privacy protection for patients will virtually limit the ability of intelligent robot data collection and deep learning, and become a legal obstacle to the application of patient data in artificial intelligence. Coupled with the largescale and multi-domain development of artificial intelligence, it is difficult to discover and regulate various risks before they are formed. Alternative measures, including artificial intelligence compulsory liability insurance, can, to some extent, balance the relationship between strict regulation by law and the promotion of industry prosperity. In 2016, the British Parliament proposed a proposal to extend the original compulsory insurance coverage to product liability, the policyholder determine the subject of liability for accidents according to the current Consumer Protection Law in the UK and product liability and fault principle in common law. Therefore, before the artificial intelligence putting into business, the corresponding subject is compelled to take out liability insurance, to pay out in advance of the smaller economic insurance risk sharing opportunities. This will greatly reduce the occurrence of artificial intelligence infringement of economic compensation disputes, directly promote the healthy development of the artificial intelligence industry.

\section{ThOughts on Legal FunCtion IN THE AgE OF INTELLIGENT ROBOT}

In the traditional environment, legal means are the most powerful, effective, stable and universal way to regulate lifesociety relations. However, after the rise of any technology represented by artificial intelligence, intelligent robots with deep learning characteristics have brought a lot of uncertainty [6], the law is dying?

In the future, artificial intelligence may gain complete autonomy and realize independent action strategies and moral choices without human control. This will certainly have a strong impact on the existing social order, the importance of the law will not decrease but increase. Perhaps, in order to avoid this situation, the limitation of the autonomy of artificial intelligence, so that its behavior can be expected by humans, will become the choice that humans have to make. However, the law should not sit idly by, amending the boundaries of the law, reaffirming the function of the law, and building a timely rule of law in a deep learning environment is undoubtedly a wiser choice. 


\section{CONCLUSION}

The development of artificial intelligence, not only to mention the needs in newly social life, but also push a legal evolution for the new era. The general application of artificial intelligence emphasizes the mode of knowing the law by the public, also emphasizes the formal and practical aspects of the law itself. Intelligence robots with deep learning and autonomous decision-making ability will change the rules of traditional tortious subject. To share the international experiences, strive to achieve the organic unity of preventing risks and social progress through legal coercion and social guidance. There's no doubt that the law is getting more and more important in the wholly new world.

\section{REFERENCES}

[1] Thomas Reed, the Rise of Machines-a Lost History of Cybernetics. Mechanical Industry Press, 2017.

[2] Jason, Artificial Intelligence for Health and Health Care[R] McLean VA Jason, 2017.

[3] Obermeyer Z, Emanuel E. Predicting the Future: Big Data, Machine Learning, and Clinical Medicine [J]. The New England Journal of Medicine, 2016, 375: 1216-1219.

[4] Shademan A, Decker R S, Opfermann J D, et al. Supervised Autonomous Robotic Soft Tissue Surgery[J]. Science Translational Medicine, 2016, 8(337): 337-364.

[5] Miller A. The future of health care could be elementary with Watson [J], CMAJ, 2013,185(9) .

[6] Yu Chengfeng, The Death of Law: The Crisis of Legal Function in the Age of Artificial Intelligence [J]. Journal of East China University of Political Science and Law, 2018,2:5-20.

[7] Yang Chunfu. Legal Intepretation of Social Risk Law [J]. Law and Social Development, 2011, 6:106-114. 